\title{
Language and number in Down syndrome: The complex developmental trajectory from infancy to adulthood
}

\author{
Sarah Paterson \\ Neurocognitive Development Unit, Institute of Child Health, London
}

\begin{abstract}
This paper examines language and number understanding in infants with Down syndrome and Williams syndrome and compares infant performance to that of adults. The crosssyndrome/ cross-domain studies demonstrate that the pattern of performance of infants with Down syndrome and Williams syndrome on two tasks assessing language and number cannot be derived from the pattern of proficiencies and impairments in the adult phenotypic outcome. Single word comprehension was assessed using a visual preference paradigm. All groups (Williams syndrome, Down syndrome, chronological age and mental age-matched controls) looked longer at the stimuli which matched the verbal label but the infants with Down syndrome and Williams syndrome were equally delayed (equivalent to their mental age controls). The similarity between the infants with Down syndrome and those with Williams syndrome did not parallel the difference present in the adult phenotypes, where vocabulary skill in Down syndrome is significantly lower than that in Williams syndrome. Number was assessed using a novelty preference paradigm, in which infants were familiarised with displays of 2 objects and then presented with 2 versus 3 objects. Infants with Williams syndrome discriminated between the familiar and novel numerosities. Infants with Down syndrome did not. Again, the difference between the Down syndrome and Williams syndrome infant groups did not parallel the pattern seen in the adult phenotypes, where individuals with Down syndrome performed better than those with Williams syndrome. It is therefore crucial to characterise the infant state, in order to understand fully the developmental trajectories of atypical groups.
\end{abstract}

Keywords - vocabulary comprehension, number skills, Down syndrome, Williams syndrome, developmental trajectories, infancy, adulthood, cognitive development.

\section{Introduction}

The cognitive phenotypes of neurodevelopmental disorders, such as Down syndrome and Williams syndrome, have been characterised in a snap-shot fashion at various points later in development. However, very few studies have attempted to chart their developmental trajectories and trace them back to infancy. The research presented in this paper aims to take a truly developmental approach to the cognitive profile of these atypically developing groups: one that is well-known and highly researched, Down syndrome, and the other that is much rarer, Williams syndrome. Instead of merely characterising abilities in the endstate when the phenotype has reached maturity, the research attempts to make links between this state and the nature of specific abilities and impairments at the outset of development, in infancy. Only when the infant state is fully characterised can we begin to understand the effects of development itself on disorders such as Down syndrome and Williams syndrome (Karmiloff-Smith, 1998; Paterson, Brown, Gsödl, Johnson \& Karmiloff-Smith, 1999). Ultimately, it will be necessary to chart the full developmental trajectories of these disorders to ascertain how they differ from each other and from the typical pattern.

The aim of this research is to investigate the language and numerical abilities in infancy and in adulthood of two atypically developing groups. The syndromes were chosen because they exhibit a pattern of distinctive cognitive phenotypes in later life, with both differences and similarities in the steady state. Williams syndrome has a very uneven cognitive profile with marked strengths and weaknesses. Similarly, peaks and troughs of ability are present in Down syndrome, although the profile is less extreme.

Down syndrome is more common than Williams syndrome, occurring in 1 in 600-800 births. It usually results from an additional copy of chromosome 21 , the most common of which is trisomy 21 . In order to reduce variability, all participants in this research have the trisomy 21 type. Down syndrome gives rise to clear physical characteristics and learning disabilities, with IQs in a similar range to those found in Williams syndrome, generally in the $50 \mathrm{~s}-60 \mathrm{~s}$ range. Previous work suggests that the pattern of strengths and weaknesses in the Down syndrome cognitive profile are almost the opposite of those found in Williams syn- 
drome. Individuals with Down syndrome have particular difficulties with language and with number, but are less impaired on tasks that assess spatial skills (e.g. Klein \& Mervis, 1999).

Williams syndrome is a much less common neurodevelopmental disorder caused by a submicroscopic deletion on chromosome $7 \mathrm{qll}, 23$. It occurs in approximately 1 in 20,000 live births and its clinical features include several physical abnormalities accompanied by mild to moderate mental retardation and a specific personality profile. Like Down syndrome, IQs are generally in the 50 s and 60 s. The cognitive profile of the mature Williams syndrome phenotype is characterised by marked strengths and weaknesses. Several studies have suggested that despite general cognitive impairment, face processing and vocabulary skills are relatively spared, whereas number and visuo-spatial constructive ability is poor (e.g. Bellugi, Wang \& Jernigan, 1994; Mervis, Morris, Bertrand \& Robinson, 1999).

In addition to the two clinical groups, two control groups were formed: a mental-age-matched group, to give an indication of typical performance at a given developmental level, and a chronological-age-matched group, to take into account length of experience. For this research, mental age was obtained from a widely used, standardised infant ability test, the Mental Scale of the Bayley Scales of Infant Development II (Bayley, 1993). Adults were matched using mental age equivalents from the British Abilities Scale (Elliott, 1996).

Williams syndrome, with its particularly uneven pattern of strengths and weaknesses, has been used by some theorists to support claims about innate and independently functioning modules (e.g. Pinker, 1999). Several researchers believe that the static neuropsychological model derived from data from adults with brain damage can be applied to developmental disorders (Baron-Cohen, 1998; Leslie, 1992; Temple, 1997). Their argumentation implies that patterns of strengths and weaknesses in the mature phenotype are necessarily present in the initial state. For example, Williams syndrome is seen as having an intact language module, an intact face processing module and an impaired spatial cognition module, with relative strengths seen as intact rather than merely less impaired (Pinker, 1994; 1999). Given the pattern reported for Williams syndrome and Down syndrome, this view would predict that infants with Down syndrome would have impaired language and number and that infants with Williams syndrome would have intact language but impaired number. However, this adult model may not be appropriate for neurodevelopmental disorders which develop differently from the outset (Karmiloff-Smith, 1998).

This paper attempts to illustrate that individuals with Down syndrome and Williams syndrome show different patterns of development in infancy than in adulthood. It is inappropriate to consider their impairments in terms of missing or intact modules, but instead they should be thought of as the product of different developmental trajectories (Elman et al., 1996; Karmiloff-Smith, Brown, Grice \& Paterson, in press; Paterson et al., 1999). It is likely that the developing organism does not have pre-specified modules but instead has mechanisms which are more suited to particular types of inputs and processing than others. These biases in mechanisms lead to their progressive specialisation as a product of development (Elman et al., 1996; KarmiloffSmith, 1998). By this view, it is unlikely that impairments or enhancement of behaviour can be linked directly to specific genes. Instead, in atypical development, genetic abnormalities will lead to subtle changes in the developmental trajectory of individuals and that this in turn will alter the processes which bring about the gradual specialisation of cognitive function.

The above discussion highlights how crucial it is to examine cognitive ability from the very outset of development, to investigate whether differences in performance occur as a product of different developmental processes, affecting learning and the processing of inputs from the environment, or whether the differences in neurodevelopmental disorders are already present at birth.

In order to apply a developmental approach, two aspects of cognitive development in Down syndrome and Williams syndrome were examined both in infancy and in the phenotypic outcome, although of course in long-term research it will be essential to chart the developmental trajectory between the two. The domains of number and language were chosen because language abilities differ substantially in the two syndromes in the mature phenotype, but number abilities are reported to be seriously impaired in both groups. This difference in outcomes, both within and across syndromes, allows a number of important theoretical and practical issues to be addressed. One can ask if the pattern of abilities present in adulthood can be used to infer the pattern seen in infancy or if individuals with a neurodevelopmental disorder such as Down syndrome follow a completely different developmental trajectory.

Studies investigating these two domains are reported in this paper. The first is an examination of receptive vocabulary. A study with infants with Down syndrome and Williams syndrome is reported in detail followed by a summary of a similar study conducted with older children and adults. The second study is an investigation of numerosity discrimination in infancy and is also followed by a report on an adult study. The results of these studies will be discussed in terms of their implications for both theory and practice. 


\section{Study I: Receptive vocabulary in infancy and adulthood}

\section{I.a. Infant Study}

\section{Method}

\section{Participants}

Data were collected from 71 infants: 22 with Down syndrome matched with 15 with Williams syndrome, 17 mental age-matched controls (matched using mental ages from the Bayley Scales of Infant Development II) and 17 chronological age-matched controls.

See Table 1 for mean mental and chronological ages for each group.

\section{Apparatus and stimuli}

A preferential looking paradigm based on Golinkoff, HirshPasek, Cauley and Gordon (1987) was used. Each infant was presented with 16 trials made up of a pair of images, each seen twice.

Infants were tested using a replica of the basic Fagan apparatus (Fagan, 1970). This apparatus is a portable visualpreference viewing box, which allows the presentation of two visual stimuli simultaneously. It has a hinged display panel ( $85 \mathrm{~cm}$ long and $29 \mathrm{~cm}$ high), with two slots to hold the stimulus cards. In this study, the stage was illuminated using a fluorescent lamp, which was out of the infant's view. The position of the lighting enabled the experimenter to see the infants' pupils clearly. The centre-to-centre distance between the slots was $30.5 \mathrm{~cm}$, and on all trials, the display stage was situated approximately $30.5 \mathrm{~cm}$ above the infant's head. In the centre of the stage was a peephole $0.625 \mathrm{~cm}$ in diameter, through which an observer, blind to the position of the stimuli, could see the visual fixations of the infant. This was done by looking for the reflection of the stimulus on which the infant fixated, in her pupil. Infants were tested in a special infant seat in order to keep them still and in the correct position.

The stimuli were coloured photographs mounted onto white cards 17.7 x $17.7 \mathrm{~cm}$. Images of 16 familiar objects were chosen and their size, brightness and complexity were controlled for. The following objects were used: cup, clock, table, house, spoon, phone, teddy, doll, ball, socks, shoes, hat, cat, dog, duck and fish. Stimuli were presented in pairs that were randomly generated. Every pair was seen twice, with the position of the cards changed on each occasion.

\section{Procedure}

The child was settled into the infant seat and then the testing apparatus was wheeled into position, with the peephole in the display stage centred directly over the infant. During this time, the experimenter spoke to the infant, who could no longer see the parent. The stimuli were then placed into the two slots simultaneously by the experimenter. Once the infant's attention was obtained by talking or by shaking a rattle, the presentation began. Just before presenting the display to the infant, i.e., just as the flap was closing, the experimenter began the linguistic input. In a loud, clear voice, using the highly pitched intonation of "motherese" she said, "Look, look at the X" (the named stimulus) and repeated this three times while the stimuli were on display. Each infant was shown 16 trials. Each trial consisted of the presentation of two pictures. Each pair was presented twice, once with the named item on the left and again later in the sequence with the named item on the right, or vice versa. The side on which the named object appeared was randomised. A second experimenter held a stopwatch in each hand and timed the infant's looking to the left versus the right stimulus item by observing the corneal reflection of each stimulus in the infant's pupil. Reliability using this procedure has been shown to be high (Haaf, Brewster, de Saint Victor \& Smith, 1989; O’Neill, Jacobson \& Jacobson, 1994).

A timer, which was set to five seconds, signalled when a trial was to end. Between each familiarisation trial, the experimenter pulled back the flap from the infant's view, recorded the times called out by the observer, and changed the stimulus cards. Once the infant's gaze was centred and her attention obtained, the flap was closed exposing the next stimulus to the infant.

\section{Results}

Cumulative looking-time to each picture was recorded by the experimenter. Only data from pairs in which the child was familiar with both objects, as assessed by parents before the test, were included in the analysis. This was done following a problem with the Golinkoff et al. (1987) study. Golinkoff and her colleagues point out that if an infant is presented with two images, e.g. sock and hat, and is familiar with only one of them (sock), he might look at the item corresponding to the verbal input (hat), simply because he recognises that a sock is not a hat. The number of items included in the analysis varied from 2 pairs to all 16 pairs, depending on the parental report. If an infant had formed a link between a spoken word and its referent, one would expect that she would look longer at the picture that matched the label which she heard than at the non-matching picture. Analyses were carried out to ascertain whether infants in each group exhibited this understanding.

Not all infants were included in the analyses. Outliers were found using

Table I. Mean chronological and mental ages for each group. 
a box and whisker plot. The main body of the plot represented values between the 25 th and 75 th percentile. Participants whose looking-times were more than 1.5 box lengths above or below these values were excluded from the analysis, according to the instructions provided in the SPSS statistical package. This made very little difference to the numbers in each group. One infant was removed from both the Williams syndrome and mental age-control groups and two were removed from the Down syndrome group. There were no outliers in the chronological age-control group.

A repeated measures Anova with group as the within-subjects variable and match or non-match as the between-subjects variables, was carried out to search for differences in mean looking-times across subjects. The mean lookintimes to matching and non-matching stimuli by group are shown in Figure 1. There was a significant difference in looking time to matching and non-matching stimuli, $F$ (1, $64)=87.89, p<.0001$ with longer looks to the matching stimulus. No effect of group emerged, $F(3,64)=1.90$, n.s. However, there was a significant interaction between group and match, $F(3,64)=6.64, p<.001$. Post hoc t-tests examining the differences within each group between lookingtimes to the matching and non-matching stimuli revealed significant differences in all groups, but to a lesser extent in the Down syndrome group (Williams syndrome $t(14)=$ $4.65, p<.0001$; Down syndrome $t(20)=3.21, p<.004$; mental age-control $t(15)=4.06, p<.001$; chronological age-control $t(16)=6.41, p<.0001)$.

\section{I.b. Adult study - vocabulary}

The receptive vocabulary of 7 adults with Down syndrome (mean chronological age 22, range 9;11-33;7 / mean mental age $5 ; 9$, range $5 ; 1-6 ; 4$ ) and 7 adults with Williams syndrome was assessed (mean chronological age $21 ; 6$, range $9 ; 10-33 ; 4 /$ mean mental age $6 ; 9$, range $5 ; 1-9 ; 4)$. The groups were matched on chronological age and on mental

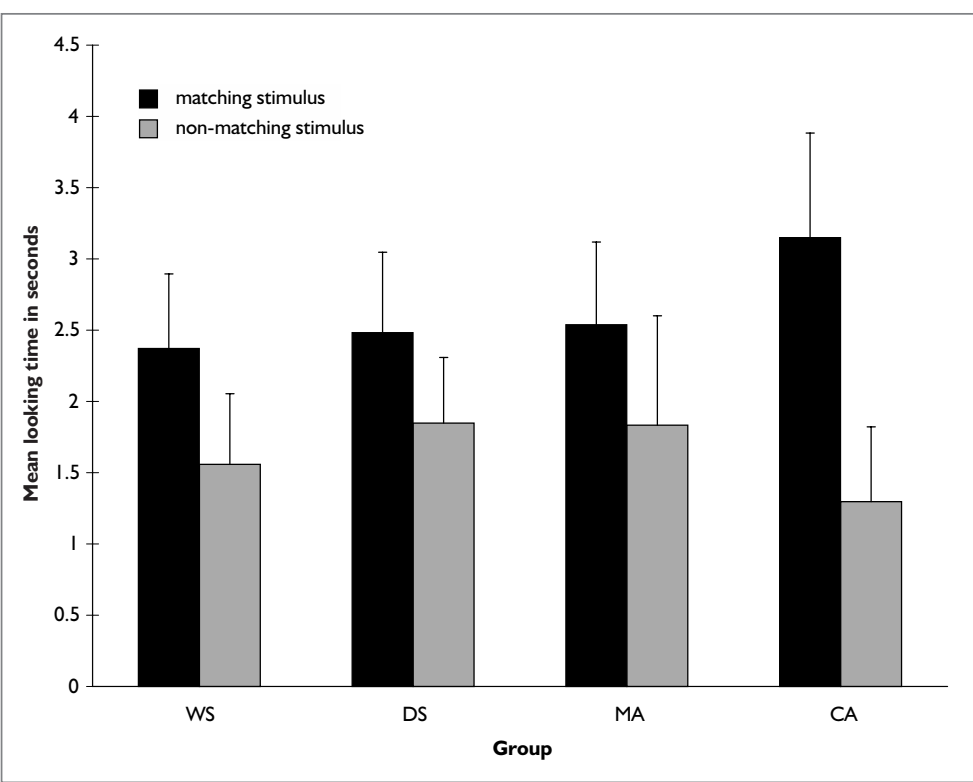

Figure I. Mean looking-times to matching and non-matching stimuli in seconds. age from the British Ability Scales $(t(13)=2.05, p>.06)$. Vocabulary knowledge was ascertained using the British Picture Vocabulary Scale (BPVS; Dunn, Dunn \& Whetton, 1982), a standardised test of receptive vocabulary. The test age of each participant was then compared with chronological age. There was a smaller discrepancy between chronological age and test age on the BPVS for adults with Williams syndrome than for those with Down syndrome, $t(6)=2.55, p<.05$, suggesting that the individuals with Down syndrome had a greater vocabulary impairment than those with Williams syndrome.

\section{Study I - Discussion}

Despite marked differences in later language ability, both the infants with Down syndrome and those with Williams syndrome were equally able to match a word to its referent in the early stages of development, i.e. 24-36 months. It should be noted, however, that both groups were equally delayed in language acquisition (Paterson, 2000). The pattern of their looks is more like that seen in the mental age-matched controls than that of children of the same chronological age. In addition, on a standardised parental assessment of vocabulary (MacArthur Communicative Development Inventory, Fenson et al., 1993) both the Down syndrome and Williams syndrome participants had smaller receptive vocabularies than controls. This highlights the importance of studying language ability in infancy, particularly in clinical groups.

The pattern of language abilities seen in the Down syndrome and Williams syndrome groups in this infant study is not the same as that seen in adulthood. This important fact would have been missed if infant performance had been simply inferred from adult data, because the adult data suggest that vocabulary comprehension in the individuals with Williams syndrome would be higher than that present in their counterparts with Down syndrome. The adult data also give little indication of the degree of delay in both groups.

\section{Study 2: Numerosity comprehension}

\section{2.a. Infant study}

\section{Method}

\section{Participants}

Sixty-five infants took part in this experiment. Twenty-two infants with Down syndrome were tested, along with 13 infants with Williams syndrome, matched on both chronological age and mental age, 16 mental-age matched typically developing infants and 14 chronologicalage matched typically developing infants. The mean chronological and mental ages of each group are presented in Table 2 , and indicate a good match. 


\begin{tabular}{|llccccc|}
\hline & $\begin{array}{l}\text { Mean CA } \\
\text { (months) }\end{array}$ & SD & $\begin{array}{l}\text { Range } \\
\text { (months) }\end{array}$ & $\begin{array}{l}\text { Mean MA } \\
\text { (months) }\end{array}$ & SD & $\begin{array}{l}\text { Range } \\
\text { (months) }\end{array}$ \\
\hline WS & 30.5 & 5.62 & $12-24$ & 16.9 & 2.81 & $12-21$ \\
DS & 29.5 & 4.90 & $12-24$ & 15.6 & 2.43 & $12-20$ \\
MA-matched & 15.4 & 2.52 & $12-20$ & 15.1 & 2.66 & $11-21$ \\
CA-matched & 30 & 5.30 & $12-24$ & 30.4 & 5.32 & $25-40$ \\
\hline
\end{tabular}

Table 2. Mean chronological and mental ages for each group. were calculated for each infant. A box and whisker plot of the data from each group revealed outliers in some groups. This meant that data from one infant with Williams syndrome, two infants with Down syndrome and from one mental age-matched typically developing infant were not included in the analysis. The remaining data points were entered into a repeated measures

\section{Apparatus and stimuli}

As in Study 1, infants were tested using a replica of the basic Fagan apparatus (Fagan, 1970). Stimuli for this experiment consisted of six white cards 17.7 x $17.7 \mathrm{~cm}$. Coloured pictures of two objects were mounted on each card in different positions. The objects depicted differed on each card and included aeroplanes, cats, dogs, cars etc. The test cards both depicted new objects with one displaying 2 items, the other 3 items. The objects on each card were of different shapes and sizes and in different positions on the card. This was done so that infants could not use merely the area of the card covered to determine which displayed the novel numerosity.

\section{Procedure}

The test procedure was based on that used by Starkey, Spelke and Gelman (1990) with typically developing infants. Infants were familiarised with the pairs of stimuli depicting arrays of 2 objects in different configurations and then tested with the display of 2 versus 3 objects. The stimuli were placed into the two compartments simultaneously by Experimenter I and, once the infant's attention was attained by talking or by shaking a rattle, the familiarisation trials began. Each infant was shown 6 familiarisation trials. After familiarisation with sets of 2 , the infant was presented simultaneously with one card displaying new objects but the old numerosity (2) and one displaying new objects but a novel numerosity (3). The side on which the novel numerosity appeared was randomised and Experimenter II, who measured the cumulative looking time over each trial, was blind to the position of that card. A beeper was set to a fixed length for the familiarisation and test trials, and signalled when a trial was to end (10 seconds for familiarisation, 5 seconds for test).

\section{Results}

It was predicted that if infants were merely sensitive to the novelty of the objects, they would show no preferential looking because all arrays contained novel objects. However, if they had become sensitive to the constant numerosity of 2 in the displays in the familiarisation phase, then they should look significantly longer at the new numerosity of 3 in the test phase.

The mean looking-times to the novel ( 3 pictures) and familiar (2 pictures) numerosities

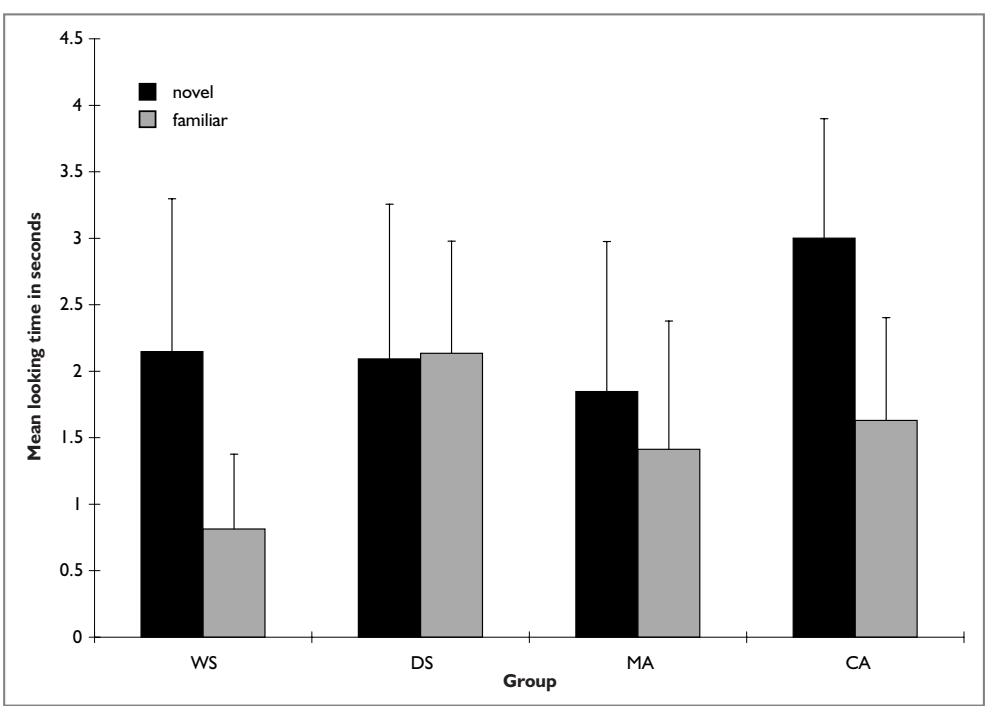

Figure 2. Mean looking-times to novel and familiar numerosities in seconds
Anova with group as the between-subjects factor (Down syndrome, Williams syndrome, mental age-matched, chronological age-matched) and numerosity (novel or familiar) as the within-subjects factor. An effect of numerosity $F(1$, $57)=34.21, p<.001$ and of group $F(3,57)=5.94, p<.001$ was found. There was also a significant interaction of group by numerosity $F(3,57)=5.94, p<.001$. This suggests that looking time to each type of stimuli (novel or familiar) differed depending on the group. In order to investigate where the differences in performance resided, post hoc t-tests were carried out. These t-tests comparing the mean scores for novel and old numerosities for each group revealed a significant difference in looking time between numerosities for the Williams syndrome, chronological age-matched and mental age-matched groups $(t=4.2$, df $11, p<.001, t=3.87$, df $13, p<.002 \& t=2.18, d f 14, p<.05$, respectively). However, there was no such difference for the Down syndrome group. The results thus show that despite their impairment in number in the endstate, in infancy participants with Williams syndrome display performance that looks like their mental-age matched and chronological-aged matched counterparts. By contrast, the group with Down syndrome showed no discrimination of the difference between the novel and old numerosities. Results for the four groups of infants are presented in Figure 2. 


\section{2.b. Adult study - numerosity}

A broad study investigating numerosity discrimination was also carried out with older children and adults (Paterson, 2000). Data from one of the experiments examining discrimination of displays made up of random dot patterns will be presented here.

Nine older children and adults with Down syndrome (mean chronological age $24 ; 3$, range $11 ; 4-35 ; 3$ / mean mental age $5 ; 9$, range $5 ; 1-6 ; 4$ ) and 8 with Williams syndrome (mean chronological age $20 ; 9$, range $10 ; 11-32 ; 9 /$ mean mental age $6 ; 9$, range $5 ; 1-9 ; 4$ ) took part. Numerosity discrimination was assessed using a well-tested paradigm which elicits the symbolic distance effect (Dehaene, Dupoux \& Mehler, 1990; Moyer \& Landauer, 1967). Numerosity judgment tasks had not been hitherto used with adults with either Down syndrome or Williams syndrome.

Participants were required to judge which of two numbers (in this case canonical dot displays) presented on a computer screen was the larger. Reaction times and accuracy were measured. In the typical case, a distance effect is always apparent: numbers very close (like 7 and 8 ) take longer for a decision as to which is the larger than numbers that are far apart (like 7 and 2).

The results of this study demonstrated that adults with Down syndrome and Williams syndrome performed differently on the numerosity judgement tasks. The adults with Down syndrome, although slower overall, showed a clearcut distance effect as evidenced by typically developing controls. By contrast, the adults with Williams syndrome performed significantly worse than the matched adults with Down syndrome on several number tasks and, although there was a trend in the right direction, the Williams syndrome group did not show a robust distance effect. There was a significant difference between the Down syndrome and Williams syndrome groups for the discrepancy between reaction times to close and far pairs for comparison of canonically spaced dot displays (Mann-Whitney $U=-11$, $p<.05$ )

While designing this research, an attempt was made to devise similar number tasks with adults and toddlers. In both cases, the tasks involved making a comparison between two numerosities. In the toddler case, an implicit same/different judgement was elicited, whereas for the adults an explicit decision was required as which of the numerosities was the larger. The processes used in the toddler task involve similar comparisons and are likely to be a foundation of those used in the adult task, calling on basic representations of numerosity in the brain.

\section{Study 2 - Discussion}

The study described above provided the first data ever to characterise the performance of infants with Down syndrome and Williams syndrome using a numerosity discrimination task. Typically developing infants, whether mental age or chronological age-matched, looked significantly longer at the arrays of a novel numerosity (3) after familiarisation with arrays of 2 . The infants with Williams syndrome performed like their typically developing counterparts, whereas the infants with Down syndrome did not. This is interesting, given the impaired pattern of abilities reported for these atypically developing groups in older childhood and adulthood (Bellugi, Bihrle, Neville, Doherty \& Jernigan, 1992; Nye, Clibbens \& Bird, 1995; Sloper, Cunningham, Turner \& Knussen, 1990) and the results from a study of numerosity discrimination reported above.

On the basis of the data, it seems again that performance in the steady states of late childhood and adulthood is not reflected in the abilities of atypically developing infants. While the group of infants with Down syndrome appears to have impairment in a basic ability which underlies later number ability, the infants with Williams syndrome do not, despite more serious problems later with numerical tasks. This means that the developmental trajectory for number understanding in these two clinical groups differs. In Down syndrome, problems with number appear to reside in its foundations and are apparent very early in development, causing considerable delay. By contrast, in Williams syndrome it seems that at least one of the foundations of number understanding is functioning normally. Either the root of later problems in this group occurs further along the developmental pathway, or alternatively another of the fundamental building blocks for number may not function appropriately. Further research following the same infants longitudinally over time should make it possible to discover when the number problems begin and in which tasks they are encountered.

\section{General discussion}

The results from Studies 1 and 2 suggest that the trajectories in developmental disorders are complex and that the infant cognitive profile cannot be inferred from that present in adulthood, as has been done often in the past. In the number domain we demonstrated that in infancy individuals with Down syndrome cannot discriminate between small numerosities but that in adulthood they are able to make discriminations and exhibit robust distance effects. The opposite is true for Williams syndrome, in which infants are able to discriminate numerosities but adults do not perform as well as those with Down syndrome and exhibit a much weaker distance effect. In the language domain the receptive vocabulary of groups are delayed in infancy but in adulthood individuals with Williams syndrome exhibit a clear advantage other those with Down syndrome. These results have implications for both theory and practice.

\section{Theoretical implications}

In the past, many researchers have used the pattern of strengths and weaknesses present in older children and adults with developmental disorders to infer the pattern of abilities present in infancy. In addition, in typical development the pattern in the endstate has been used to make arguments about the innateness of certain abilities. Until 
recently, researchers inclined to posit the existence of innate principles in various cognitive domains, for example, in theory of mind or number, have tended to assume that these principles were generally similar to and operated in much the same manner as adult principles (Leslie, 1992; Wynn, 1992). In autism, for example, Leslie (1992, 1994) argues for the existence of a theory-of-mind module. He shows that older children and adults with autism are poor at understanding false beliefs in social situations but are successful in understanding "false" outcomes in physical situations, such as out of date representations like photographs. Leslie takes impairments which were found in older children and adults and uses these to posit the existence of an innate module. This assumes that the impairments are due to the same cognitive module being defective in infancy. However, Leslie has shown only that the endstate, when all the crucial development has already taken place, is impaired.

Dissociations in abilities across development from infancy to adulthood, such as those reported in this paper suggest that such a static approach to atypical development is unwise. The results suggest that neurodevelopmental disorders should not be thought of in terms of impaired and intact cognitive modules, present at birth. Instead, the data highlight that very different outcomes can arise from similar starting states (as illustrated by the vocabulary data). It is also possible that different starting states can result in similar outcomes. Atypically developing infants with different syndromes follow different developmental trajectories, so it is crucial to study the process of development and not merely to make assumptions using data from the endstate.

\section{Practical implications}

The results have important implications for the type and timing of interventions used with individuals with Down syndrome. It is likely to be more effective to provide support and training as early as possible in development to re-route the developmental trajectory of a particular skill towards the typical trajectory. For example, given the data which suggest that adults with Down syndrome perform well on tasks assessing numerosity discrimination, those who take the static view of development might argue that intervention in the domain of number would not be useful. However, in infancy the foundations of this ability were shown not to be present. Thus, early intervention, during the period of maximal cortical plasticity, aimed at strengthening representations of numerosity might have a positive effect on more general number development and lead to even better performance later on. In the case of Williams syndrome, knowledge about the relatively good receptive vocabulary of adults might lead one to suspect that vocabulary intervention is unnecessary, but from the infant data it is clear these individuals have delayed language acquisition and would benefit from early linguistic intervention.

In sum, then, the pattern of abilities seen in infants with Down syndrome and Williams syndrome cannot necessarily be inferred from the pattern seen in adulthood. Instead, it appears that these different neurodevelopmental disorders follow different developmental trajectories which must be traced all the way from infancy to adulthood in future research.

\section{Acknowledgements}

This research was presented at the $3^{\text {rd }}$ International Conference on Language and Cognitive Development in Down Syndrome, Portsmouth, UK. Portions of this paper first appeared in Science (Paterson et al., 1999). It was supported by a PhD studentship from the Down Syndrome Association to the first author and by Programme and Project grants from the Medical Research Council to A. Karmiloff-Smith.

\section{Correspondence}

Dr Sarah Paterson - Neurocognitive Development Unit, Institute of Child Health, 30 Guilford Street, London, WCIN IEH.• E-mail: s.paterson@ich.ucl.ac.uk

\section{References}

Baron-Cohen, S. (1998). Modularity in developmental cognitive neuropsychology: Evidence from autism and Gilles de la Tourette syndrome. In J.A. Burack, R.M. Hodapp and E. Zigler (Eds.) Handbook of Mental Retardation and Development. Cambridge, UK: Cambridge University Press.

Bayley, N. (1993). Bayley Scales of Infant Development: Second Edition. San Antonio, TX: The Psychological Corporation.

Bellugi, U., Bihrle, A., Neville, H., Doherty, S., \& Jernigan, T. (1992). Language, cognition, and brain organization in a neurodevelopmental disorder. In M. Gunnar \& C. Nelson (Eds.), Developmental Behavioural Neuroscience (pp. 201-232). Hillsdale, NJ: Lawrence Erlbaum.

Bellugi, U., Wang, P. P., \& Jernigan, T. L. (1994). Williams syndrome: An unusual neuropsychological profile. In $S$. H. Broman \& J. Grafman (Eds.), Atypical Cognitive Deficits in Developmental Disorders: Implications for Brain Function (pp. 23-56). Hillsdale, NJ: Lawrence Erlbaum.

Dehaene, S., Dupoux, E., \& Mehler, J. (1990). Is numerical comparison digital? Analogical and symbolic effects in two-digit number comparison. Journal of Experimental Psychology: Human Perception and Performance, 16(3), 626-641.

Dunn, L.M., Dunn, L.M. \& Whetton, C. (1982) British Picture Vocabulary Scale. Windsor, Berks.: NFER Nelson.

Elliott, C.D., Smith, P. \& McCulloch, K. (1996). British Ability Scales II. Windsor, Berks: NFER-Nelson.

Elman, J., L., Bates, E., A., Johnson, M., H., KarmiloffSmith, A., Parisi, D. \& Plunkett, K. (1996). Rethinking Innateness: A Connectionist Perspective on Development. Cambridge, MA, USA: The Mit Press.

Fagan, J.F. (1970). Memory in the infant. Journal of Experimental Child Psychology, 9 (2), 217-226.

Fenson, L., Dale, P.S., Reznick, J.S., Thal, D.J., Bates, E., Hartung, J.P., Pethick, S.J. \& Reilly, J.S.(1993). MacArthur Communicative Development Inventories. San Diego, CA: Singular Publishing Group. 
Golinkoff, R. M., Hirsh-Pasek, K., Cauley, K. M., \& Gordon, L. (1987). The eyes have it: Lexical and syntactic comprehension in a new paradigm. Journal of Child Language, 14, 23-45.

Haaf, R. A., Brewster, M., de Saint Victor, C. M., \& Smith, P. H. (1989). Observer accuracy and observer agreement in the measurement of visual fixation with fixed-trial procedures. Infant Behaviour and Development, 12 (2), 211-220.

Karmiloff-Smith, A. (1998). Development itself is the key to understanding developmental disorders. Trends in Cognitive Sciences, 2(10), 389-398.

Karmiloff-Smith, A., Brown, J.H., Grice, S. \& Paterson, S.J. (in press). Dethroning the myth: Cognitive dissociations and innate modularity in Williams syndrome. Developmental Neuropsychology.

Klein, B.P. \& Mervis, C.B. (1999). Contrasting patterns of cognitive abilities of 9- and 10-year-olds with Williams Syndrome or Down Syndrome. Developmental Neuropsychology, 16 (2), 177-196.

Leslie, A.M. (1992). Pretence, autism, and the theory-ofmind-module. Current Directions in Psychological Science, 1, 18-21.

Leslie, A.M. (1994). Pretending and believing: Issues in the theory of ToMM, Cognition, 50, 211-238.

Mervis, C. B., Morris, C. A., Bertrand, J., \& Robinson, B. F. (1999). Williams syndrome: Findings from an integrated program of research. In H. Tager-Flusberg (Ed.), Neurodevelopmental Disorders: Contributions to a New Framework from the Cognitive Neurosciences. (pp. 65-110). Cambridge MA: MIT Press.

Moyer, R. S., \& Landauer, T. K. (1967). Time required for judgements of numerical inequality. Nature, 215(5109), 1519-1520.

Nye, J., Clibbens, J., \& Bird, G. (1995). Numerical ability, general ability and language in children with Down's Syndrome. Down's Syndrome: Research and Practice, 3(3), 92-103.

O’Neill, J. M., Jacobson, S. W., \& Jacobson, J. L. (1994). Evidence of observer reliability for the Fagan Test of Infant Intelligence (FTII). Infant Behaviour and Development, 17 (4), 465-469.

Paterson, S. (2000) The development of language and number understanding in Williams syndrome and Down syndrome: Evidence from the infant and mature phenotypes. Unpublished doctoral thesis, University College London.

Paterson, S., Brown, J., Gsödl, M., Johnson, M. \& KarmiloffSmith, A. (1999). Cognitive modularity and genetic disorders. Science, 286 (5448), 2355-2358.

Pinker, S. (1991). Rules of language. Science, 253 (5019), 530-535

Pinker, S. (1994). The Language Instinct. New York, NY: William Morrow \& Co.

Pinker, S. (1999) Words and Rules. London: Weidenfield \& Nicolson.

Sloper, P., Cunningham, C., Turner, S. \& Knussen, C. (1990). Factors related to the academic attainments of children with Down's syndrome. British Journal of Educational Psychology, 60, 284-298.

Starkey, P., Spelke, E. S., \& Gelman, R. (1990). Numerical abstraction by human infants. Cognition, 36(2), 97-127.
Temple, C.M. (1997). Cognitive neuropsychology and its application to children. Journal of Child Psychology \& Psychiatry and Allied Disciplines, 38 (1), 27- 52.

Wynn, K. (1992). Addition and subtraction by human infants. Nature, 358(6389), 749-750. 